\title{
A NOTE ON ARCS OF FINITE CYCLIC ORDER ${ }^{1}$
}

\author{
S. B. JACKSON
}

1. Definitions and statement of theorem. An arc $A$ in the conformal plane means the locally topological image of the closed unit interval, and small letters $p, q$, etc. will be used to denote points of $A$ or equivalently corresponding points of the parameter interval. An arc without double points is called a simple arc. If $C$ is a circle and $p \in A \cap C$ is an interior point of $A$ then $C$ is said to support [intersect] $A$ at $p$ if there is a neighborhood of $p$ on $A$ such that the two halfneighborhoods determined by $p$ lie in the same component [different components] of the complement of $C$.

Arc $A$ is said to have finite cyclic order $k$ if $k$ is the maximum number of common points of $A$ with any circle.

A circle $C$ is called a general tangent circle to $\operatorname{arc} A$ at a point $p \in A$ provided there exist triples of distinct points $\left(r_{i}, r_{i}^{\prime}, R_{i}\right)$ with sequences $\left\{r_{i}\right\}$ and $\left\{r_{i}^{\prime}\right\}$ converging on $A$ to $p,\left\{R_{i}\right\}$ converging to some point, and such that $C=\lim C\left(r_{i}, r_{i}^{\prime}, R_{i}\right)$, where $C\left(r_{i}, r_{i}^{\prime}, R_{i}\right)$ denotes the unique circle through these distinct points.

The definitions and notations above are those of Lane and Scherk [2]. The purpose of this note is to establish the following result.

TheOREM 1.1. If $A$ is a simple arc of finite cyclic order $k$ and $C$ is a circle meeting $A$ in $k$ points, then (a) $C$ intersects $A$ at any common interior point, and (b) $C$ is not a general tangent circle to $A$ at any common point.

It is to be noted that the theorem involves no smoothness assumptions on $A$ except as these are implied by the finite cyclic order. It is well known, however, that the hypothesis implies conformal differentiability at the endpoints and hence at any interior point one-sided conformal differentiability with respect to both adjoining arcs $[1 ; 2 ; 3]$.

2. Proof of part (a) of the theorem. Let $q_{i}, i=1, \cdots, k$, be the points of $A \cap C$ taken in order on $A$, and let $A^{\prime}$ be the subarc of $A$ with endpoints $q_{1}$ and $q_{k}$. Arc $A^{\prime}$ clearly also has cyclic order $k$. Let $C$ be oriented and let $Q_{1}$ and $a_{2}$ denote respectively the directed arcs $q_{1} q_{k}$ and $q_{k} q_{1}$ of $C$. Let $l_{j}\left[r_{j}\right], j=1,2$, be the number of points of

Received by the editors July 18, 1960.

1 This research was done with partial support of a fellowship from the National Science Foundation. 
$A \cap a_{j}$ where $C$ supports $A^{\prime}$ and at which $A^{\prime}$ is locally to the left [right] of $a_{j}$, and let $i$ be the number of points of intersection of $A^{\prime}$ and $C$. Since every point of $A^{\prime} \cap C$ is an intersection, a point of support, or an endpoint, it follows that

$$
k=l_{1}+l_{2}+r_{1}+r_{2}+i+2 .
$$

Consider the pencil of circles of the first kind with fundamental points $q_{1}$ and $q_{k}$, and let $C_{1}$ be a member of this pencil arbitrarily close to $C$ but such that $a_{1}$ has been displaced to its right and hence $a_{2}$ to its left. Each of the $r_{1}$ right-hand supports on $a_{1}$ is thus replaced by at least two intersections of $A^{\prime}$ with $C_{1}$ and similarly each of the $l_{2}$ left-hand supports on $a_{2}$ is replaced by at least two intersections. Each of the $i$ intersections in $A^{\prime} \cap C$ is replaced by at least one intersection in $A^{\prime} \cap C_{1}$ and the endpoints are unaltered. Hence the number of points in $A^{\prime} \cap C_{1}$ is at least $2 r_{1}+2 l_{2}+i+2$. By definition of cyclic order this is at most $k$, whence from (2.1) it follows that $r_{1}+l_{2} \leqq l_{1}+r_{2}$. A similar argument applied to circle $C_{2}$ of the pencil displaced from $C$ in the opposite sense yields the reverse inequality, whence it follows that

$$
l_{1}+r_{2}=r_{1}+l_{2} .
$$

Consider next the pencil of circles of the second kind containing $C$ and with $q_{1}$ as fundamental point, and let $C_{3}\left[C_{4}\right]$ be a circle of this pencil arbitrarily close to $C$ but displaced to the left [right] of $C$. Each of the $l_{1}+l_{2}$ left-hand supports $\left[r_{1}+r_{2}\right.$ right-hand supports] in $A^{\prime} \cap C$ is replaced by at least two intersections in $A^{\prime} \cap C_{3}\left[A^{\prime} \cap C_{4}\right]$. For definiteness assume $A^{\prime}$ lies to the left of $C$ at $q_{k}$ so this endpoint is replaced by at least one intersection in $A^{\prime} \cap C_{3}$. Since $q_{1}$ is unaltered the number of points in $A^{\prime} \cap C_{3}$ is at least $2 l_{1}+2 l_{2}+i+2$, and since as before this cannot exceed $k$ it follows by (2.1) that $l_{1}+l_{2} \leqq r_{1}+r_{2}$. A similar argument applied to $C_{4}$ shows that $r_{1}+r_{2} \leqq l_{1}+l_{2}+1$, the difference being that the endpoint $q_{k}$ is lost in this displacement. From these inequalities it follows that either (i) $r_{1}+r_{2}=l_{1}+l_{2}$ or (ii) $r_{1}+r_{2}=l_{1}+l_{2}+1$. But case (ii) is impossible since it would follow that $\left(r_{1}+l_{2}\right)+\left(l_{1}+r_{2}\right)=2\left(l_{1}+l_{2}\right)+1$ in which the right-hand side is clearly odd while by (2.2) the left-hand side is even. It follows that

$$
l_{1}+l_{2}=r_{1}+r_{2} .
$$

Suppose now that $A^{\prime} \cap C$ actually contains at least one point of support, i.e., that not all of $r_{1}, r_{2}, l_{1}, l_{2}$ are zero. Then there is a first such support $q$ as $C$ is traced from $q_{1}$ and there exists a point $B \in C$ such that $B$ and $q_{1}$ separate $q$ on $C$ from all the other supports. Let 
directed $\operatorname{arcs} q_{1} B$ and $B q_{1}$ of $C$ be denoted by $\Theta_{1}$ and $\Theta_{2}$ respectively, and for definiteness assume that $A^{\prime}$ is to the left of $C$ at $q$. Then $q \in B_{1}$ but all $r_{1}+r_{2}$ of the right-hand supports belong to $B_{2}$. Consider the pencil of circles of the first kind with $q_{1}$ and $B$ as fundamental points, and let $C^{\prime}$ be a circle of this pencil arbitrarily close to $C$ but such that $B_{1}$ has been displaced to its left and hence $B_{2}$ to it right. The support $q$ is thus replaced by at least two intersections of $A^{\prime} \cap C^{\prime}$ and the same is true of each of the $r_{1}+r_{2}$ right-hand supports on $B_{2}$. Since $q_{1}$ is unchanged and none of the $i$ intersections is lost, the number of points in $A^{\prime} \cap C^{\prime}$ is at least $2\left(r_{1}+r_{2}+1\right)+i+1$. By (2.3) and (2.1) it follows that $2\left(r_{1}+r_{2}+1\right)+i+1=r_{1}+r_{2}+l_{1}+l_{2}+i+3=k+1$ $>k$. This is impossible since $A^{\prime}$ has cyclic order $k$. Hence $r_{1}=r_{2}$ $=l_{1}=l_{2}=0$ and $k=i+2$.

If $C$ supports $A$ at one of the endpoints of $A^{\prime}$, say $q_{k}$, an arbitrarily near circle through $q_{1}$ will replace the support at $q_{k}$ by at least two intersections. Since it still meets $A^{\prime}$ arbitrarily close to each of its $i$ intersections, the number of points in $C \cap A$ is at least $i+3=k+1>k$ which is again impossible. Hence $C$ cannot support $A$ at any common interior point. This establishes part (a) of Theorem 1.1 since $A$ has finite cyclic order and hence $C$ supports or intersects at any common interior point.

3. Proof of part (b) of the theorem. In the notation of $\$ 1$, if $C=\lim C\left(r_{i}, r_{i}^{\prime}, R_{i}\right)$ where $\left\{r_{i}\right\}$ and $\left\{r_{i}^{\prime}\right\}$ converge on $A$ to $p$ and $\left\{R_{i}\right\}$ converges, it follows that for any point $R \in C$ where $R \neq p$ $C=\lim C\left(r_{i}, r_{i}^{\prime}, R\right)$. For if $R \in C$ the sequence $\left\{R_{i}\right\}$ can be chosen, without altering the sequence of circles, so that $\left\{R_{i}\right\}$ converges to $R$. But since $R \neq p$ and $\left\{R_{i}\right\}$ converges to $R$, the angle between $C\left(r_{i}, r_{i}^{\prime}, R_{i}\right)$ and $C\left(r_{i}, r_{i}^{\prime}, R\right)$ approaches zero (cf. [2, §3.34]). Hence any limit circle of $C\left(r_{i}, r_{i}^{\prime}, R\right)$ contains $p$ and $R$ and is tangent to $C$, whence it coincides with $C$ and $C=\lim C\left(r_{i}, r_{i}^{\prime}, R\right)$ as claimed.

As in $\S 2$ let $q_{1}, \cdots, q_{k}$ be the common points of simple arc $A$ with circle $C$. Suppose that at one of these points, say $q_{j}, C$ is a general tangent circle to $A$. By definition there exist sequences $\left\{r_{i}\right\}$ and $\left\{r_{i}^{\prime}\right\}$ converging to $q_{j}$ on $A$ and convergent sequence $\left\{R_{i}\right\}$ so that $C=\lim C\left(r_{i}, r_{i}^{\prime}, R_{i}\right)$.

Consider first the case when $q_{j}$ is one of the points $q_{1}$ or $q_{k}$, and let $q$ denote the other of these points. Taking $q$ as the point $R$ above, it follows that $C=\lim C\left(r_{i}, r_{i}^{\prime}, q\right)$. By $\S 2$ each of the $k-2$ points $q_{2}, \cdots, q_{k-1}$ is an intersection of $A$ and $C$ whence, for large enough $i, A \cap C\left(r_{i}, r_{i}^{\prime}, q\right)$ has an intersection arbitrarily close to each of these $k-2$ points. Since, in addition, $A \cap C\left(r_{i}, r_{i}^{\prime}, q\right)$ contains the three 
points $r_{i}, r_{i}^{\prime}, q$, it contains at least $k+1$ points which is impossible since $A$ has cyclic order $k$. Hence $C$ cannot be a general tangent circle at $q_{1}$ or $q_{k}$.

Consider next the case when $q_{j}$ is one of the points $q_{2}, \cdots, q_{k-1}$, and let $q$ be either $q_{1}$ or $q_{k}$. As before it follows that $C=\lim C\left(r_{i}, r_{i}^{\prime}, q\right)$ and that, for sufficiently large $i, A \cap C\left(r_{i}, r_{i}^{\prime}, q\right)$ has at least one intersection arbitrarily close to each of the $k-2$ points $q_{2}, \cdots, q_{k-1}$, one of which is $q_{j}$. Since $A \cap C\left(r_{i}, r_{i}^{\prime}, q\right)$ contains at least two points arbitrarily near $q_{j}$, namely $r_{i}$ and $r_{i}^{\prime}$, and also contains $q$, it contains at least $k$ points. By $\S 2$ it follows that all the points are intersections except perhaps $q$, which may be an endpoint. Thus $r_{i}$ and $r_{i}^{\prime}$ are both intersections. But since $C$ intersects $A$ at $q_{j}$, then for large enough $i$ the number of intersections of $A$ with $C\left(r_{i}, r_{i}^{\prime}, q\right)$ in an arbitrarily small neighborhood of $q_{j}$ must be odd. Thus there is another common point of $A \cap C\left(r_{i}, r_{i}^{\prime}, q\right)$ near $q_{j}$ and hence at least $k+1$ points in all, which is a contradiction as before. This completes the proof of the theorem.

4. Consequences. Two simple consequences of Theorem 1.1 may be written at once.

CoRollary 4.1. If a simple closed curve of finite cyclic order $k$ is met in $k$ points by a circle $C$, then $C$ intersects the curve at every common point and is not a general tangent circle to it at any of them.

This is obtained at once from Theorem 1.1 by considering a subarc $A$ of the simple closed curve which contains as interior points all the common points with $C$.

CoRollary 4.2. The cyclic order of a simple arc $A$ is the same as that of the open subarc obtained by deleting the endpoints.

The case of infinite cyclic order is trivial since this cannot be changed by dropping any finite number of points. If $k$ is the finite cyclic order of $A$ and circle $C$ meets $A$ in the $k$ points $q_{1}, \cdots, q_{k}$ it follows from part (a) of the theorem that all interior common points are intersections whence any circle $C^{\prime}$ arbitrarily close to $C$ will intersect $A$ close to each of the $k-2$ points $q_{2}, \cdots, q_{k-1}$. If $C^{\prime}$ is chosen to meet the open arc $q_{1} q_{k}$ of $A$ arbitrarily close to $q_{1}$ and to $q_{k}$ it will meet this open arc $k$ times. It follows that the arc consisting of the interior points of $A$ has at least cyclic order $k$. But since the cyclic order cannot exceed that of $A$ the cyclic order is exactly $k$, as was to be shown.

The special case of this result relating to arcs of cyclic order 3 is 
discussed by Lane and Scherk $[2, \S 3.3]$ in developing the properties of such arcs.

\section{BIBLIOGRAPHY}

1. J. Hjelmslev, Introduction a la theorie des suites monotones, Sep. Académie Royale des Sciences de Danemark, Nr. 1, 1914.

2. N. Lane and P. Scherk, Characteristic and order of differentiable points in the conformal plane, Trans. Amer. Math. Soc. vol. 81 (1956) pp. 356-376.

3. A. Marchaud, Sur les continus d'ordre borne, Acta Math. vol. 55 (1930) pp. 67115.

UNIVERSITY OF MARYLAND AND

UNIVERSITY OF WASHINGTON 